\title{
Evolutions Microstructurales : Observation Directe à l'Echelle Atomique grâce à la Sonde Atomique Tomographique
}

\author{
F. Danoix, B. Deconihout, A. Bostel et D. Blavette \\ Laboratoire de Microscopie Ionique, Groupe de Métallurgie Physique, URA 808 du CNRS, Faculté des \\ Sciences, place Emile Blondel, 76821 Mont Saint Aignan cedex, France
}

\begin{abstract}
Last year, we presented a contribution to illustrate the increasing role of Atom Probe techniques in the study of solid state phase transformations in metallic materials [1]. We concluded this paper with a mention to the emergence of a new generation of atom probes, giving access to quantitative $3 \mathrm{D}$ images on the sub-nanometric scale.

In this paper, we will present the instrument of this new generation developed at the Universite de Rouen, as well as some of the most interesting results we could obtain since it started to operate last year.
\end{abstract}

\section{Introduction}

Le microscope ionique à effet de champ et la sonde atomique sont des instruments d'observation et de nanoanalyse chimique dont l'importance s'est beaucoup accrue durant les quinze dernières années. Associés systématiquement, ils sont largement utilisés en sciences des matériaux, plus particulièrement pour l'étude des alliages métalliques. Leurs principes, ainsi que les principales applications de ces techniques sont décrits abondamment dans la littérature [2]. Une toute nouvelle génération de sonde atomique est apparue dernièrement avec l'émergence des sondes atomiques tridimensionnelles [3]. Ces instruments utilisent des détecteurs à localisation spatiale, ce qui permet, en plus de connaître la nature chimique des atomes, d'en déterminer la position avec une précision de quelques dixièmes de nanomètres près. Le volume analysé peut être reconstruit en trois dimensions atome par atome.

La sonde atomique Tomographique (T.A.P.), développée au Laboratoire de Microscopie Ionique de l'Université de Rouen (U.R.A. C.N.R.S. 808) est la dernière née de cette nouvelle génération [4]. Les caractéristiques et performances de cet instrument seront présentées. Nous illustrerons ensuite ses potentialités sur quelques exemples métallurgiques choisis.

\section{Limites de la sonde atomique conventionnelle}

Dans une sonde atomique, les atomes de l'échantillon s'évaporent par effet de champ sous forme d'ions. Les ions formés sont accélérés par le champ électrique régnant autour de l'échantillon. La mesure du temps de vol des ions entre l'échantillon et un détecteur permet d'en déterminer la vitesse, donc le rapport masse sur charge, donnant ainsi accès à la nature chimique des atomes évaporés. L'interaction entre le champ électrique et l'ion en mouvement va déterminer de manière quasi-univoque la trajectoire de ce dernier. On forme ainsi une image de la surface de l'échantillon, correspondant approximativement à une projection stéréographique du réseau. La connaissance de la position de l'impact de l'ion sur un détecteur permet alors de déterminer la position qu'occupait l'atome à la surface de l'échantillon avant son départ, par simple projection inverse. 
La résolution spatiale à la surface de l'échantillon, ou résolution latérale, n'est intrinsèquement limitée que par les aberrations de trajectoire des ions lors des premiers nanomètres de vol. Les aberrations sont dues à l'environnement local immédiat de l'atome s'évaporant. Elles conduisent à une imprécision latérale de quelques dixièmes de nanomètre pour la trajectoire de l'ion par rapport à son point de départ. L'utilisation dans les sondes atomiques conventionnelles de détecteurs insensibles à la position des impacts conduit à une perte de l'information de position latérale $(\mathrm{x}, \mathrm{y})$ portée par l'ion. La résolution spatiale latérale est alors déterminée par la taille du détecteur. Cette perte transforme une analyse au départ tridimensionnelle en une analyse monodimensionnelle, de type profil de concentration selon l'axe de la pointe $(z)$. L'investigation s'interprète comme la traversée du matériau par un cylindre d'analyse dont le diamètre est la taille projetée du détecteur. L'information contenue dans ces profils de concentration est très souvent réductrice de la réalité car elle résulte de la projection d'une microstructure en trois dimensions sur un axe. Une connaissance a priori de la microstructure est très souvent nécessaire pour accéder aux véritables grandeurs (compositions, tailles, longueurs de corrélation) à partir de celles mesurées, en particulier lorsque les dimensions impliquées sont de l'ordre de grandeur de celle de la surface d'analyse, favorisant des effets complexes de convolution spatiale.

La solution retenue pour réduire au minimum les convolutions spatiales consiste à réduire la surface d'analyse (surface du détecteur projeté à la surface de l'échantillon). On se heurte alors au problème inévitable des fluctuations statistiques d'échantillonnage qui sont inversement proportionnelles au nombre d'atomes recueillis. Le risque est alors de cacher la majeure partie des informations contenues dans les profils de concentration dans un bruit de fond d'origine statistique.

\section{La Sonde Atomique Tomographique.}

Ces réflexions sur les limites des sondes conventionnelles ont conduit à l'émergence d'une nouvelle génération d'appareils, basés sur l'utilisation de détecteurs à localisation spatiale. Ils permettent de reconstituer en 3D le volume analysé atome par atome, d'où leur nom générique de sondes atomiques tridimensionnelles, avec une résolution spatiale de quelques dixièmes de nanomètres.
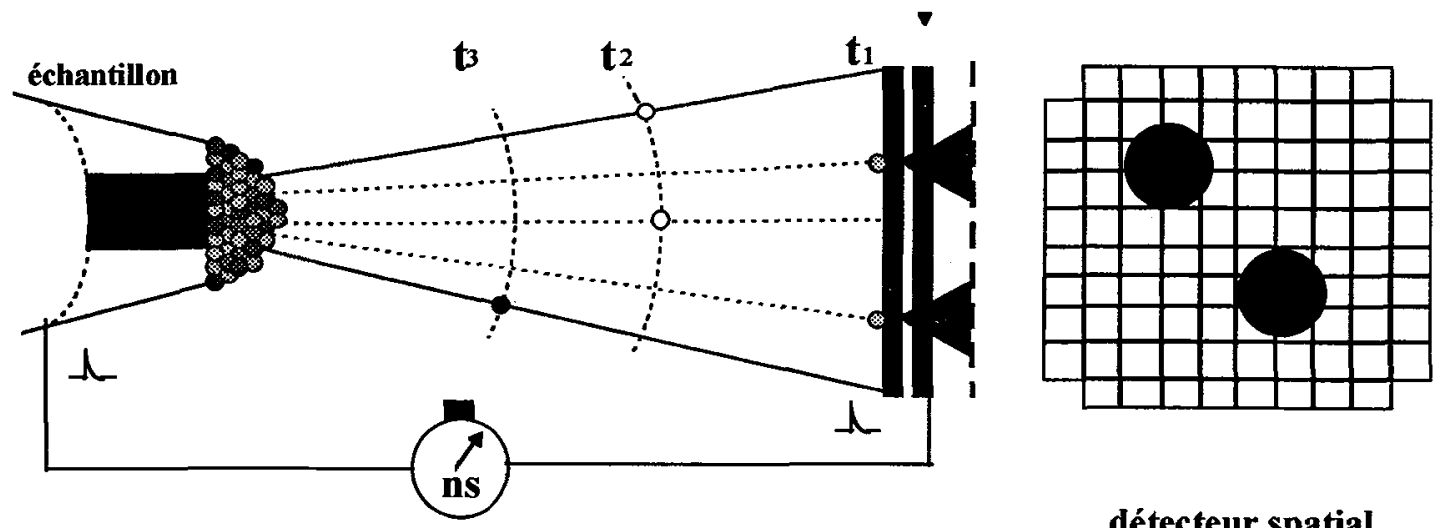

\section{détecteur spatial}

Figure 1 : schéma de principe de la Sonde Atomique Tomographique.

Les défauts et qualités des sondes 3D sont intrinsèquement liés aux propriétés du détecteur spatial bidimensionnel choisi. Une étude détaillée des différents systèmes proposés a été réalisée par Deconihout [5]. Il apparaît que des trois configurations utilisées: le POSAP (Position Sensitive Atom-Probe [3]), l'OAP (Optical Atom-Probe [6]) et le TAP (Tomographic Atom-Probe ou Sonde Atomique Tomographique [7]), cette dernière possède indiscutablement les meilleures performances. Cet avantage résulte du fait que le principe de la Sonde Atomique Tomographique repose sur l'emploi d'un multi-détecteur spatial sensible à 
des impacts multiples qu'ils soient simultanés ou pas [7]. En effet, cette condition, pour des raisons explicitées par ailleurs [5], est indispensable pour réaliser des analyses réellement quantitatives. Schématiquement, le détecteur comprend deux galettes de microcanaux (GMC) placées devant un réseau carré de $10 \times 10$ anodes indépendantes (figure 1). Chaque anode possède sa propre chaîne de mesure analogique des charges. L'arrivée d'un ion crée, à la sortie des G.M.C., un nuage d'électrons qui irradie le réseau d'anodes. La mesure de la répartition spatiale de la charge entre les quelques anodes irradiées permet de calculer la position de l'impact sur le détecteur. Chaque anode et son électronique associée étant indépendante, plusieurs impacts peuvent être localisés en même temps. En mesurant les quantités de charges recueillies sur la multianode, la position du barycentre du spot d'électrons et donc de l'impact, peut être calculée [7].

\section{Performances de la Sonde Atomique Tomographique.}

La résolution spatiale, la résolution en masse et la quantitativité des mesures sont les principaux critères permettant de juger des performances de l'appareil. C'est donc tout naturellement que nous nous intéresserons d'abord aux performances de la Sonde Atomique Tomographique relativement à ces aspects, avant d'aborder la partie consacrée à l'utilisation de la Sonde Tomographique en métallurgie physique.

\section{IV-1 La résolution spatiale}

A cause de la nature même du processus d'évaporation par effet de champ, et tout comme pour une sonde classique, la résolution spatiale se décompose en deux parties: la résolution latérale et la résolution en profondeur.

La résolution latérale de l'instrument est liée à celle du détecteur utilisé. Elle a été évaluée de manière expérimentale. Un masque, percé de trous circulaires, a été placé devant la multianode. Ces trous, dont le diamètre varie de 1.5 à $4 \mathrm{~mm}$, ont été répartis selon des cercles et des droites inclinées de manière à briser la symétrie du détecteur. L'image ionique obtenue au travers du masque est présentée figure 2 .

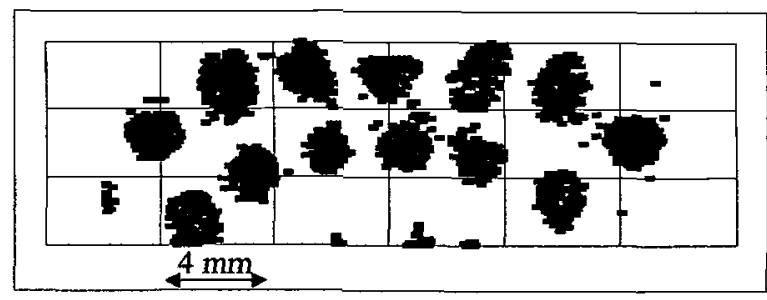

Figure 2: image ionique calculée des trous du masque ( $\phi_{\text {trous }}=2 \mathrm{~mm}$ ).

L'étude détaillée de cette image montre que les positions des impacts ioniques sont résolues avec une précision inférieure à $0.3 \mathrm{~mm}$. Le grandissement de l'instrument étant de l'ordre de $10^{7}$, une telle précision correspond à une résolution absolue à la surface de l'échantillon de $0.03 \mathrm{~nm}$. Il en résulte que la résolution latérale intrinsèque de la Sonde Tomographique est limitée pour l'essentiel, par les aberrations des trajectoires ioniques évoquées précédemment, soit quelques dixièmes de nanomètre. Il n'est à l'heure actuelle pas envisageable de reconstruire parfaitement le cristal.

Le problème de la résolution en profondeur est très différent. La surface de l'échantillon étant une sphère, elle présente à l'échelle atomique une succession de terrasses atomiques et de décrochements. Parmi toutes les terrasses, celles correspondant à l'interception des plans de bas indices de Miller ont des dimensions de quelques nanomètres. Il est possible d'orienter l'échantillon de sorte que la surface d'analyse en sonde atomique classique ne couvre qu'une seule et unique terrasse. Le matériau est alors analysé plans atomiques par plans atomiques. L'obtention de la résolution atomique en profondeur est cependant soumis à la condition que les différents plans atomiques analysés, même s'ils sont de nature chimique différente, s'évaporent successivement. Lors d'une analyse en Sonde Atomique Tomographique, l'obtention d'une telle résolution n'est pas aussi immédiate: la surface du détecteur étant 100 fois supérieure, la surface d'analyse intercepte toujours plusieurs terrasses atomiques. Les atomes détectés peuvent être issus de plans différents. La possibilité de garder une résolution en profondeur strictement égale à une couche atomique dépend de la capacité à décourber l'image ionique reçue par le détecteur. La procédure utilisée à cette fin est suffisamment performante pour atteindre cet objectif, comme le montre la figure 3 . La résolution en profondeur atteint dans ce cas strictement un plan atomique (002). Ce résultat montre que, dans la mesure 
ou l'on peut atteindre la résolution atomique en profondeur en sonde atomique classique, on peut aussi l'atteindre avec la Sonde Atomique Tomographique.

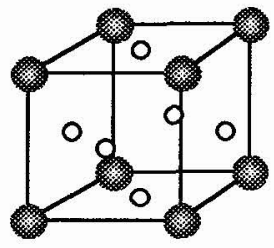

$\circ \mathrm{Ni}$

Al

\begin{abstract}
Figure 3 :Analyse ì la Sonde Atomique Tomographique dans la direction [001] d'un alliage $\mathrm{Ni} 3 \mathrm{AlSi}$ ordonné, de structure L12. L'image montre clairement la succession des plans riches puis pauvres en aluminium de la structure $\mathrm{LI}_{2}$. La distance entre plans riches en aluminium est égale à $0.36 \mathrm{~nm}$.
\end{abstract}

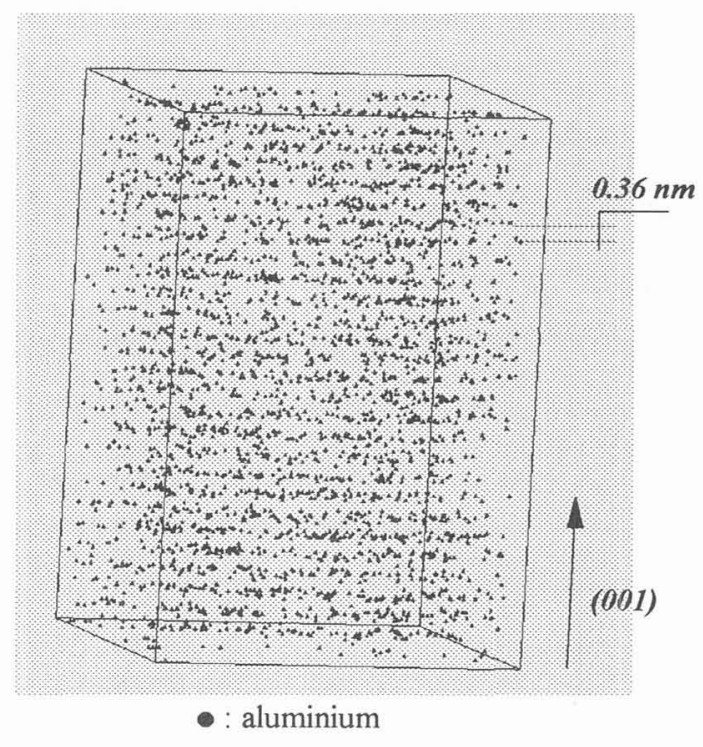

\title{
IV-2 La résolution en masse
}

La résolution en masse et la quantitativité des mesures peuvent être illustrées sur un exemple métallurgique simple. L'image tridimensionnelle présentée sur la figure 4 est le résultat de l'analyse d'un superalliage à base nickel (CMSX2). Cet alliage contient de gros précipités cuboïdaux (taille $\approx 0,45 \mu \mathrm{m})$ riches en aluminium (phase $\gamma^{*}$ ) dispersés dans une matrice riche en chrome (phase $\gamma$ ). La figure représente la distribution en trois dimensions des atomes de chrome (noir) et d'aluminium (blanc) rencontrés dans le volume analysé. Les autres éléments, dont le nickel, ne sont pas représentés. Durant l'analyse, deux particules $\gamma^{t}$ et un couloir de matrice $\gamma$ ont été traversés.

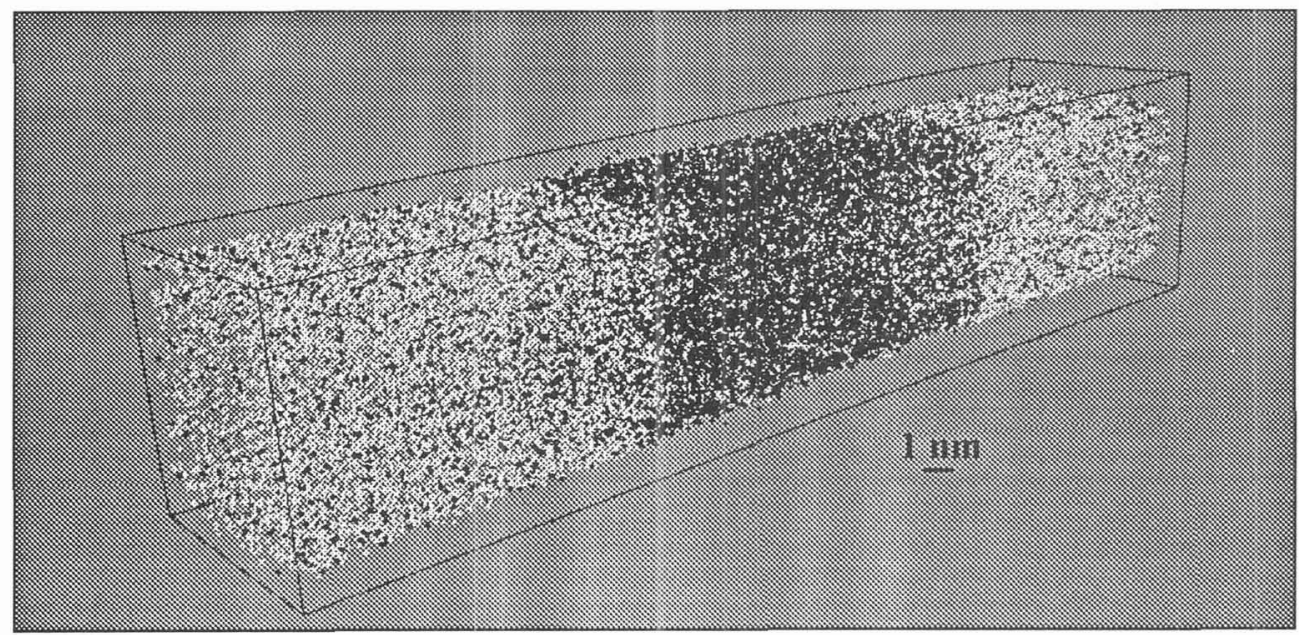

Figure 4 : distribution en volume des atomes de chrome (en noir) et d'aluminium (en blanc) dans un superalliage à base nickel CMSX2. 


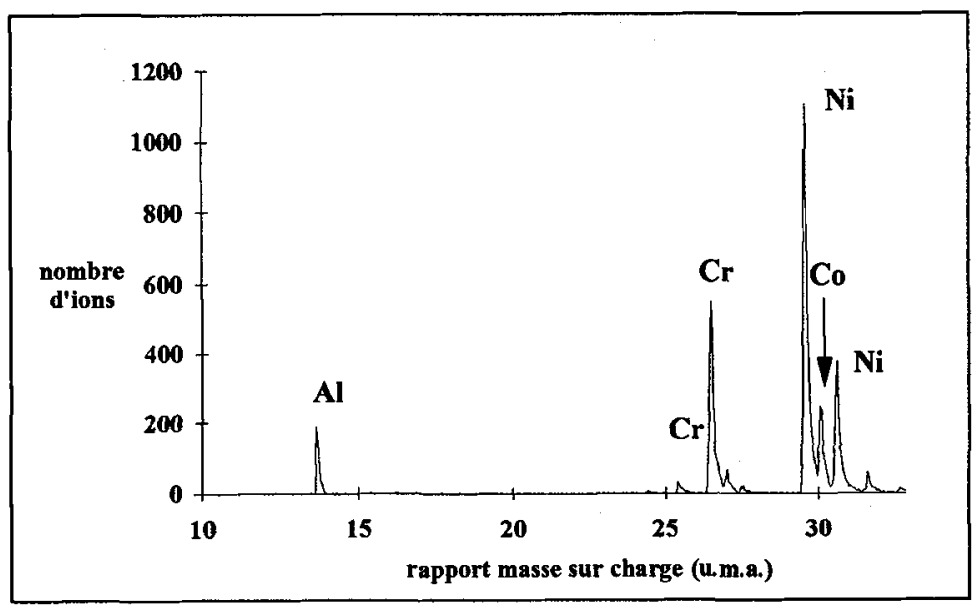

La figure 5 représente le spectre de masse relatif à l'analyse de la phase $\gamma$. La résolution en masse est définie par $R=\frac{M / n}{\Delta(M / n)}$, ou $\Delta(M / n)$ est la largeur du pic de masse.

\section{Figure 5: spectre de masse relatif $\dot{a}$ la phase $\gamma$.}

En spectrométrie de masse, $R$ est généralement appréciée à la demi hauteur des pics: $\Delta(M / n)$ est la largeur du pic mesurée à la moitié de sa hauteur. La résolution à mi-hauteur vaut ici $\mathrm{R}_{50 \%}=200$. Cependant, les pics de masse obtenus sont dissymétriques, de sorte que la résolution à mi-hauteur est peu représentative de la qualité des spectres de masse et en particulier de leur largeur à la base. On préfère souvent à $\mathrm{R}_{50 \%}$, la résolution $\mathrm{R}_{10 \%}$ mesurée à $10 \%$ de la hauteur des pics. La résolution $\mathrm{R}_{10 \%}$ mesurée est égale à 60 . Dans les deux cas, cette résolution est comparable à celle obtenue avec une sonde classique.

\section{IV-3 La quantitativité des mesures}

Le rendement de détection d'une sonde atomique est environ 0.6: ceci résulte de l'utilisation des galettes de microcanaux, dont la surface sensible est de $60 \%$. Pour assurer la quantitativité des mesures, il faut que le rendement de détection ne dépende pas de la nature chimique de l'ion détecté, ce que l'expérience a montré pour les sondes classiques [2]. Les galettes de microcanaux étant aussi à la base du détecteur de la Sonde Atomique Tomographique, on peut s'attendre à ce que ce soit aussi le cas pour cet instrument. Le tableau 1 compare les compositions mesurées dans la phase $\gamma^{\prime}$ d'un superallige à base nickel à celles précédemment mesurées en sonde classique. Les valeurs sont très voisines. On vérifie ainsi qu'il est possible d'obtenir des mesures quantitatives de composition avec la Sonde Atomique Tomographique. Notons que pour une acquisition de durée comparable, 100 fois plus d'atomes sont collectés en Sonde Tomographique, la statistique sur les mesures des concentrations s'en trouve donc améliorée d'un facteur 10.

\begin{tabular}{|c|c|c|}
\hline élément & $\begin{array}{c}\text { concentration } \\
(\text { at.\% } \% 2 \sigma)\end{array}$ & $\begin{array}{c}\text { concentration } \\
\text { attendue [8] }\end{array}$ \\
\hline $\mathrm{Al}$ & $17.4 \pm 0.9$ & 16.5 \\
\hline $\mathrm{Ti}$ & $2.1 \pm 0.4$ & 1.7 \\
\hline $\mathrm{Cr}$ & $3 \pm 0.4$ & 3.8 \\
\hline Autres (Ni, Co ...) & $77.5 \pm 1$ & 78 \\
\hline
\end{tabular}

\section{Tableau 1 : composition de la phase $\gamma^{\prime}$ d'un superalliage à base nickel (CMSX2) mesurée à la Sonde Atomique Tomographique.}

Il faut cependant signaler une situation où la quantitativité des mesures peut être affectée par l'analyse en Sonde Tomographique. Il s'agit de l'analyse en mode plan atomique par plan atomique. Lorsque le centre d'une terrasse fini de s'évaporer, il peut arriver que plusieurs atomes de même nature chimique quittent simultanement la pointe, saturant le multidétecteur. La localisation de ces atomes est impossible, et des atomes sont perdus sélectivement (aussi bien en terme de nature chimique que d'origine sur la pointe). L'analyse de ce genre d'expérience est, du point de vue des mesures de composition, plus délicate, car le biais expérimental peut varier en fonction du site observé sur une reconstruction. 


\section{Etude des transformations de phase à l'aide de la Sonde Atomique Tomographique}

Nous allons maintenant illustrer les performances et potentialités de ce nouvel instrument en Sciences des Matériaux au travers de quelques résultats. Nous montrerons par la même occasion, comment les inconvénients des sondes atomiques classiques, ont, pour une large part, été dépassés par l'utilisation d'une sonde tridimensionnelle.

\section{V-1 Analyse d'un ioint de grains.}

Le premier exemple permet d'illustrer la puissance de la Sonde Atomique Tomographique dans un cas simple: l'étude de la répartition des solutés autour d'une interface plane: un joint de grains. Le matériau analysé est un superalliage à base nickel ASTROLOY dopé au bore [9]. Si l'ajout de bore améliore de façon sensible les propriétés mécaniques de ce type d'alliage, les phénomènes qui régissent ce changement de comportement mécanique sont encore mal connus. Des travaux en cours au laboratoire, montrent que certains éléments ségrègent aux joints de grains. Leur concentration au coeur du joint est un des paramètres critiques dans l'explication des changements de propriétés mécaniques. La mesure de cette concentration à l'aide de la sonde classique s'avère délicate. En effet, lorsque le joint de grains n'est pas perpendiculaire à la direction d'analyse, l'épaisseur ségrégée est artificiellement accrue, et il est difficile d'accéder à sa composition sans faire appel à des procédures de déconvolution spatiale complexes.

Dans le cas de l'analyse en Sonde Atomique Tomographique, l'orientation du joint n'a aucune importance. L'image donne une vision directe de la distribution spatiale des différentes espèces chimiques présentes au voisinage du joint. La figure 6 représente les distributions 3D des différents éléments rencontrés dans le volume analysé [10]. On distingue nettement la présence d'un joint de grains au centre de l'image incliné à $45^{\circ}$ environ par rapport à la direction d'analyse. La zone enrichie en bore s'étend sur environ $3 \mathrm{~nm}$. Il convient cependant d'être prudent quant à l'interprétation de cette épaisseur. Les aberrations dans la trajectoire des ions de bore peuvent conduire à un élargissement artificiel de la zone enrichie. Ce point est discuté dans un article récent [11]. Les atomes de bore ne sont pas tous confinés dans le joint ; une traîne apparaît dans chacune des phases. De plus, on remarque un appauvrissement en Al+Ti.

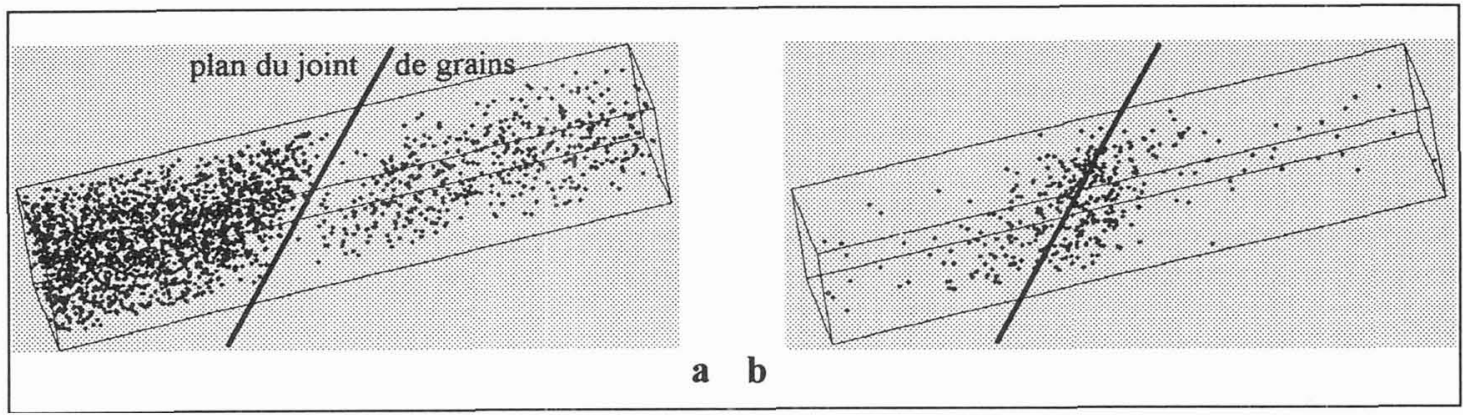

\section{Figure 6 : analyse d'un joint de grains. Répartition tridimensionnelle des atomes de $A I+T i(a)$ et $B+C$ (b). (Résultats L. Bazile-Letellier)}

Le résultat d'une analyse en sonde atomique conventionnelle correspondrait au profil tracé le long de la direction d'analyse présenté figure 7-a. On y mesure une teneur en bore au joint de grain environ égale à $12 \%$ at. Or l'image du volume analysé montre que le joint est incliné par rapport à la direction d'analyse: le profil de concentration ne reflète donc pas la composition du seul joint.

La Sonde Atomique Tomographique permet de reconstituer le volume analysé. Il est donc possible d'accéder à une analyse dans la direction la plus favorable à une analyse quantitative: la direction perpendiculaire au plan du joint. Le profil résultant d'une telle analyse est montré figure 7-b. On voit que la concentration en bore est en fait proche de $20 \%$.

De plus, le nombre d'atomes collectés par unité de volume est 50 fois supérieur à celui obtenu en sonde classique: l'incertitude des mesures de composition (inversement proportionnelle à la racine carrée de ce nombre) est donc grandement réduit. 


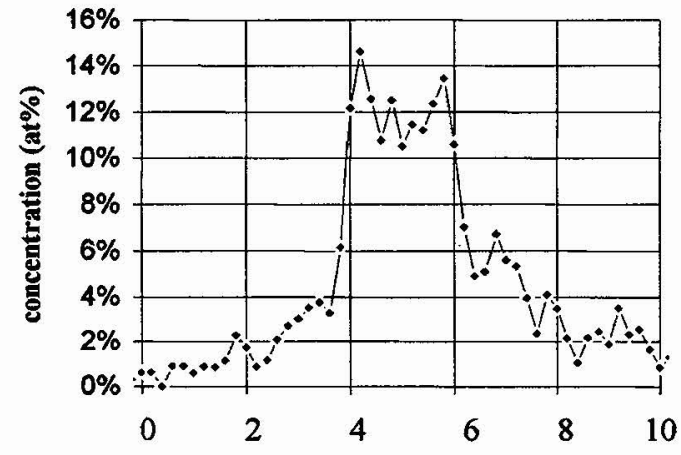

distance en nanomètres

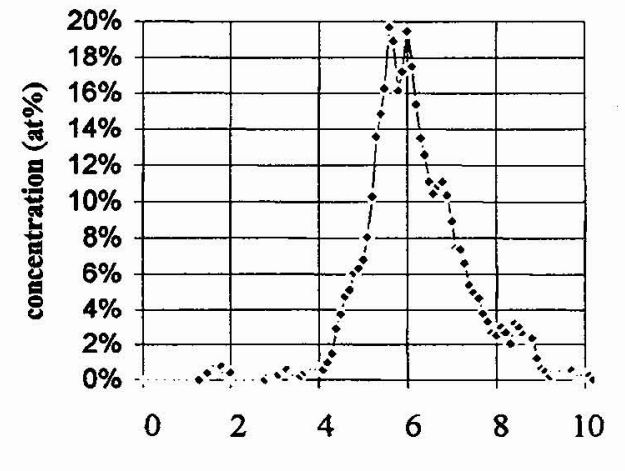

distance en nanomètres

Figure 7 : profils de concentration tracés le long de la direction d'analyse (a) et perpendiculairement au plan du joint de grains (b).

\section{V-2 Analyse de petites particules finement dispersées}

L'analyse de systèmes décomposés présentant de petites particules finement dispersées est possible en

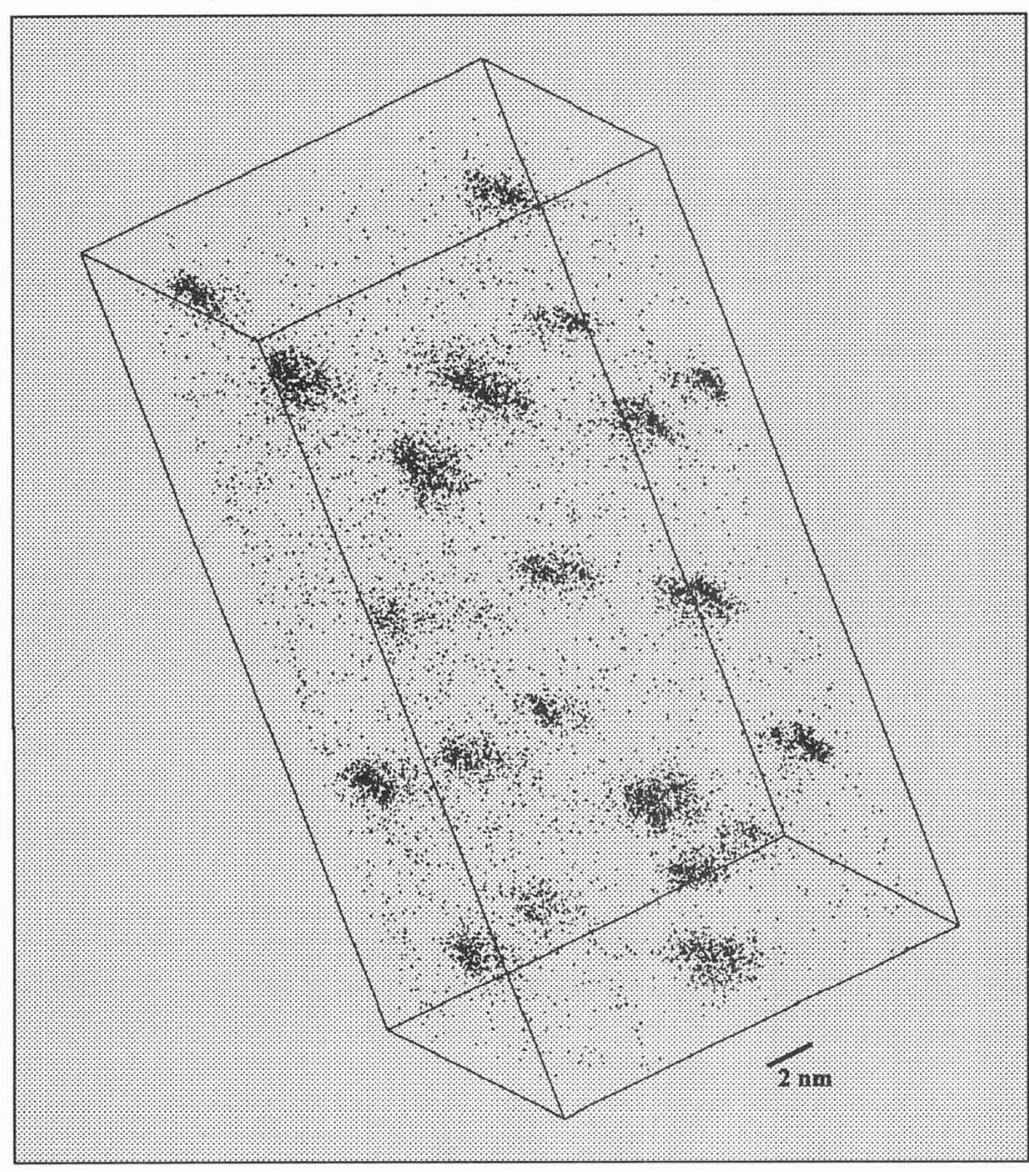
sonde atomique classique mais suppose un traitement statistique des profils de concentration afin d'obtenir la taille et la composition des particules, classique nécessite une hypothèse cruciale: celle de l'homogénéité en forme, taille et composition des particules analysées, ce qui n'est généralement ainsi que la fraction volumique précipitée [12]. Cependant le traitement des profils obtenus en sonde pas le cas.

Figure 8: Distribution tridimensionnelle des atomes de cuivre dans un alliage $\mathrm{Fe}-1,4 \%$ at Cu irradié aux neutrons -d'après [13] (Résultats P PAREIGE) 
La Sonde Atomique Tomographique, quant à elle, donne directement limage tridimensionnelle de la répartition des atomes dans l'espace. On peut alors exploiter les résultats obtenus sans hypothèse à priori sur la nature des objets contenus dans le volume analysé. La figure 8 présente par exemple le résultat d'une analyse d'un alliage $\mathrm{Fe}-1,4 \%$ at $\mathrm{Cu}$ dont l'irradiation aux neutrons favorise la formation de petites particules enrichies en cuivre. L'image tridimensionnelle présentée montre la distribution des atomes de cuivre dans le volume analysé. Une vingtaine de particules riches en cuivre, et dont la taille est infërieure ou égale à $2 \mathrm{~nm}$, sont visibles. Certaines informations essentielles sont directement accessibles à partir d'une telle reconstruction. Ce sont par exemple la taille des particules (ainsi que la distribution), leur forme (ici proche d'une ellipsoïde) ainsi que le nombre de particules par unité de volume. Un traitement plus spécifique permet d'obtenir la composition de chacune de ces particules.

\section{V-3 Décomposition spinodale de la ferrite dans un acier duplex inoxydable.}

La phase ferritique riche en chrome des aciers inoxydables austéno-ferritiques se décompose, selon un processus spinodal isotrope lorsqu'elle est vieille aux environ de $300-400^{\circ} \mathrm{C}$. Deux domaines se forment, l'un riche en chrome $\left(\alpha^{\prime}\right)$, l'autre en fer $(\alpha)$, par augmentation de l'amplitude des fluctuations de concentrations à mesure que l'acier vieillit. Les deux domaines $\alpha$ et $\alpha$ ' forment deux réseaux continus complémentaires et interconnectés. La topologie de la phase ferritique vieillie est complexe, elle présente une structure percolée de type "éponge". Ce type de structure, par nature tridimensionnelle, ne présente pas de symétrie simple. On comprend aisément que, de part son caractère unidimensionnelle, l'analyse en sonde atomique classique empêche de caractériser complètement la topologie complexe engendrée par cette décomposition spinodale [14]. Seules l'amplitude moyenne et les longueurs d'ondes de ces fluctuations sont accessibles à partir des profils de concentration. Des paramètres importants, tels que son degré de connectivité ou sa dimension fractale, deviennent accessibles avec l'analyse tridimensionnelle du matériau.

La figure 9 représente la distribution en volume des atomes de chrome observée à la Sonde Atomique Tomographique. On voit clairement limage en trois dimensions de cette structure dont on mesure la complexité. L'exploitation de ce genre d'image nécessite bien sûr le développement de nouveaux concepts et de nouveaux outils aptes à caractériser et à quantifier les propriétés tridimensionnelles de cette structure. Des travaux récents ont d'ailleurs permis d'avancer dans ce domaine [15].

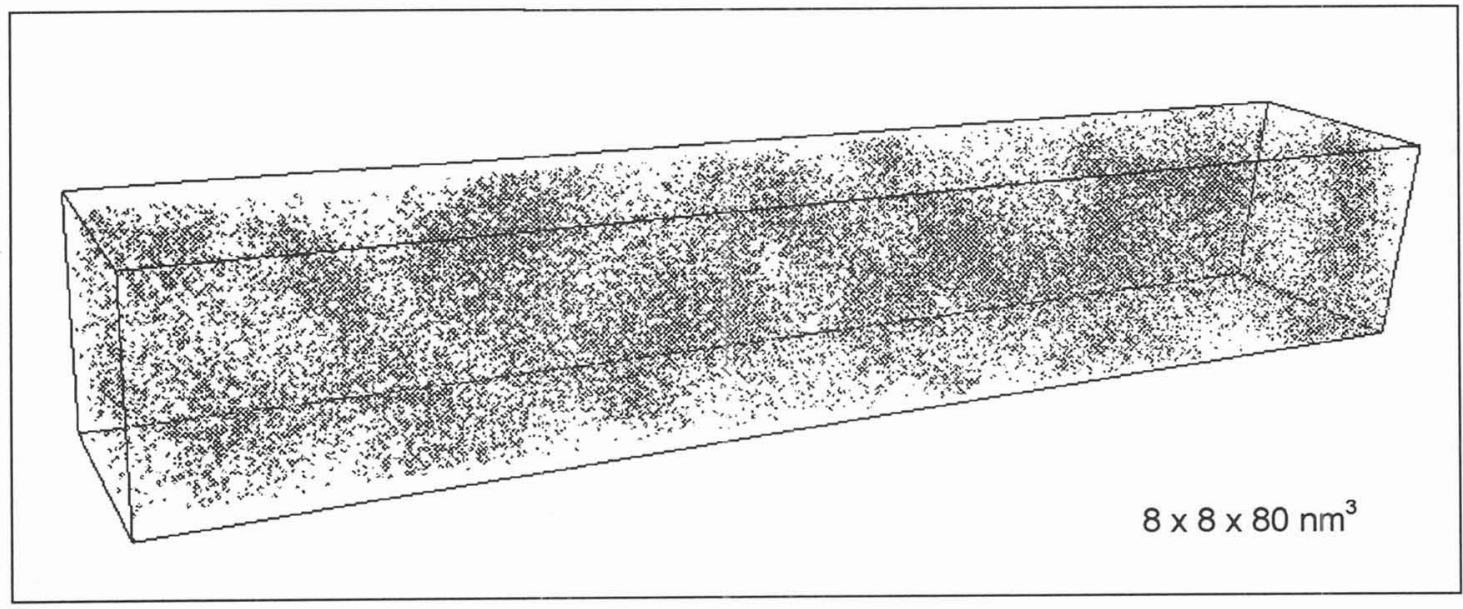

\section{Figure 9 : Répartition des atomes de chrome dans la phase ferritique d'un acier duplex vieilli 30000 heures à $400^{\circ} \mathrm{C}$.}

\section{V-4 Etude des cinétiques de mise en ordre}

Comme nous l'avons montré au chapitre IV-1, la résolution en profondeur de la Sonde Tomographique est strictement égale à un plan atomique. Nous avions illustré ce résultat par l'analyse, 
dans une direction [001], d'un matériau ordonné de structure $\mathrm{Ll}_{2}$. On est donc en mesure de discriminer les plans atomiques successifs perpendiculaires à la direction d'analyse. Ceci n'est plus le cas dans les autres directions. En d'autres termes, la résolution latérale dans un plan perpendiculaire à la direction d'analyse, bien qu'elle atteigne quelques dixièmes de nanomètres, n'est pas suffisante pour permettre de replacer les atomes en coïncidence avec les différents sites atomiques. Ce résultat peut être illustré très simplement de la manière suivante. La figure 9-a représente un réseau cubique de type CFC projeté sur le plan de base (001). La figure 9-b représente ce même réseau dans lequel les atomes sont déplacés, de manière aléatoire, de $2 \AA$ autour de leur position théorique. On voit qu'une imprécision de seulement $2 \AA$ suffit à brouiller complètement l'image ordonnée.

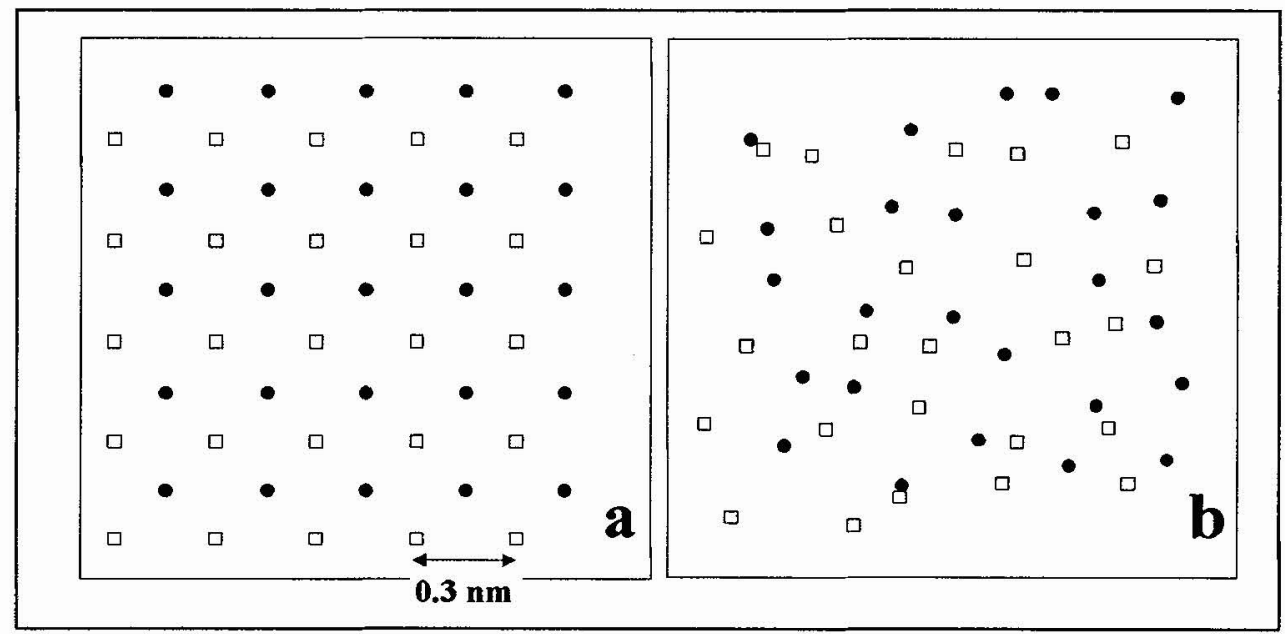

Figure 10 : influence des aberrations des trajectoires ioniques sur l'image d'un matériau ordonné.

On ne peut done strictement envisager l'étude en trois dimensions des cinétiques de mise en ordre, par exemple l'évolution du taux d'occupation de chaque type de sites atomiques par une espèce donnée. Il reste cependant possible de repérer les domaines ordonnés au sein d'une solution solide aléatoire, comme le montre la figure 11. On peut alors étudier l'évolution de la taille et de la morphologie des domaines ordonnés, et comparer ces résultats avec ceux obtenus par simulation numérique.

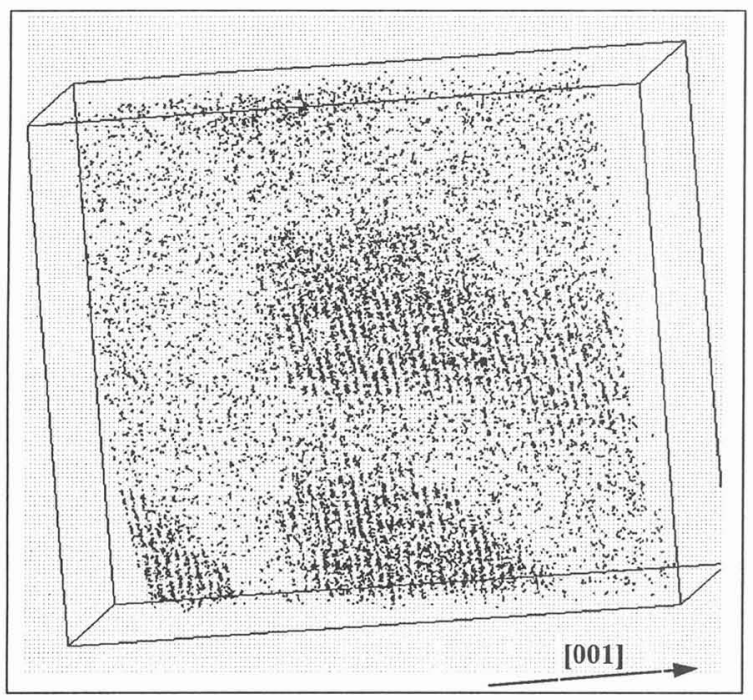

Figure 11: Petits précipités $\gamma$ de trempe dans un alliage d̀ base nickel CMSX2. Ces précipités ont une structure ordonnée de type $\mathrm{L12}_{2}$ (Ni3AI). Sur cette vue dans une direction perpendiculaire $\dot{a}$ la direction de surstructure $<001\rangle$, seuls l'aluminium est représenté. L'alternance de plans (001) riches en aluminium avec des plans pauvres en cet élément est caractéristique de la structure ordonnée des précipités.

(Résultats S. Duval) 


\section{Conclusion}

Le développement des sondes atomiques tridimensionnelles marque une étape décisive dans le développement des instruments d'analyse à très haute résolution spatiale. Le progrès réalisé par rapport à la sonde atomique conventionnelle est à la fois qualitatif et quantitatif. Qualitatif d'abord, avec l'apport et la richesse des images tridimensionnelles. Quantitatif ensuite, avec la quantité beaucoup plus importante d'informations recueillies. Une sonde $3 \mathrm{D}$ comme la Sonde Atomique Tomographique est équivalente à 100 sondes conventionnelles en parallèle si l'on considère le nombre d'ions collectés ou le volume analysé. Un demi million d'atomes est collecté en quelques heures alors qu'il fallait de nombreux échantillons et plusieurs semaines d'analyse en sonde conventionnelle pour obtenir le même résultat. Le fait le plus important n'est cependant pas cette amélioration quantitative, c'est l'obtention d'une image analytique tridimensionnelle, complètement quantitative, du matériau avec une résolution spatiale de quelques dixièmes de nanomètres. Contrairement aux techniques de diffusion des rayonnements ( $R X$, neutrons) dont l'interprétation des spectres nécessite la connaissance à priori de la composition de ces particules et de leur géométrie, on accède directement à ces informations. C'est ainsi une nouvelle approche de la microscopie et de la inicroanalyse à l'échelle atomique qui nait et ouvre de nouvelles perspectives en sciences des matériaux métalliques.

\section{Remerciements}

Les auteurs voudraient remercier Madame Bazile-Letellier ainsi que Messieurs Pareige et Duval pour leur avoir permis d'utiliser une partie de leurs travaux non publiés.

\section{Bibliographie}

[1] D. Blavette, F. Danoix, P. Auger

Journal de Physique IV, volume 4, Colloque n³, Suppl. J.P. III, n² (1994), p 35-40

[2] M.K. Miller, G.D.W. Smith

Atom-Probe microanalysis: principle and applications to materials problems, Mat. Res. Soc. Editor Pittsburg (Pa) (1989)

[3] A. Cerezo, T. J. Godfrey, G. D. W. Smith

J. de Phys., 49-C6 (1988), p 25-30

[4] D. Blavette, A. Bostel, J.M. Sarrau, B. Deconihout, A. Menand

Nature, vol 363 (1993), p 432-434

[5] B. Deconihout

Thèse de doctorat, Université de Rouen, Décembre 1993

[6] M.K. Miller

Surface Science 246 (1991), p 428-433.

[7] D. Blavette, B. Deconihout, A. Bostel, J.M. Sarrau, M. Bouet, A. Menand

Rev. Sci. Instrum. 64 (1993), p 2911-2919

[8] D. Blavette, A. Bostel

Acta Metall. vol. 32 (1984), p 811-816

[9] A. Buchon

Thèse de doctorat, Université de Rouen, Septembre 1991

[10] L. Letellier, A. Bostel, D. Blavette

Scripta Met, 30 (1994), p 1503-1508

[11] L. Letellier, M. Guttmann, D. Blavette

Phil. Mag. Letters, 70 (1994), p 189-194

[12] D. Blavette, G. Grancher, A. Bostel

J. de Phys., 49-C6 (1988), p 439-445

[13] P. Auger, P. Pareige, M. Akamatsu, D. Blavette

Journal of Nuclear Materials, 211 (1994), 194-201

[14] F. Danoix, P. Auger, A. Bostel, D. Blavette Surface Science, 246 (1991), p 260-265

[15] J.M. Hyde, A. Cerezo, M.K. Miller, G.D.W. Smith Applied Surface Science, 76/77 (1994), p 233-241 